\title{
Resuscitation of the patient with suspected/ /confirmed COVID-19 when wearing personal protective equipment: A randomized multicenter crossover simulation trial
}

\author{
Marek Malysz ${ }^{1}$, Marek Dabrowski ${ }^{2}$, Bernd W. Böttiger ${ }^{3}$, Jacek Smereka ${ }^{1,4}$, \\ Klaudia Kulak ${ }^{5}$, Agnieszka Szarpak ${ }^{6}$, Milosz Jaguszewski ${ }^{7}$, \\ Krzysztof J. Filipiak ${ }^{8}$, Jerzy R. Ladny ${ }^{1,9}$, Kurt Ruetzler ${ }^{10}$, Lukasz Szarpak ${ }^{1,5}$ \\ ${ }^{1}$ Polish Society of Disaster Medicine, Warsaw, Poland \\ ${ }^{2}$ Chair and Department of Medical Education, Poznan University of Medical Sciences, Poznan, Poland \\ ${ }^{3}$ Department of Anesthesiology and Intensive Care Medicine, University Hospital of Cologne, Köln, Germany \\ ${ }^{4}$ Department of Emergency Medical Service, Wroclaw Medical University, Wroclaw, Poland \\ ${ }^{5}$ Lazarski University, Warsaw, Poland \\ ${ }^{6}$ Maria Skłodowska-Curie Warsaw University, Warsaw, Poland \\ ${ }^{7} 1^{\text {st }}$ Department of Cardiology, Medical University of Gdansk, Poland \\ ${ }^{8} 1^{\text {st }}$ Chair and Department of Cardiology, Medical University of Warsaw, Poland \\ ${ }^{9}$ Clinic of Emergency Medicine, Medical University of Bialystok, Poland \\ ${ }^{10}$ Departments of General Anesthesiology and Outcomes Research, Cleveland Clinic, \\ Anesthesiology Institute, Cleveland, $\mathrm{OH}$, United States
}

\section{This paper was guest edited by Prof. Łukasz K. Czyżewski}

\begin{abstract}
Background: The aim of the study was to evaluate various methods of chest compressions in patients with suspected/confirmed SARS-CoV-2 infection conducted by medical students wearing full personal protective equipment (PPE) for aerosol generating procedures (AGP).

Methods: This was prospective, randomized, multicenter, single-blinded, crossover simulation trial. Thirty-five medical students after an advanced cardiovascular life support course, which included performing 2-min continuous chest compression scenarios using three methods: (A) manual chest compression (CC), (B) compression with CPRMeter, $(C)$ compression with LifeLine ARM device. During resuscitation they are wearing full personal protective equipment for aerosol generating procedures. Results: The median chest compression depth using manual CC, CPRMeter and LifeLine ARM varied and amounted to 40 (38-45) vs. 45 (40-50) vs. 51 (50-52) mm, respectively $(p=0.002)$. The median chest compression rate was 109 (IQR; 102-131) compressions per minute (CPM) for manual CC, 107 (105-127) CPM for CPRMeter, and 102 (101-102) CPM for LifeLine ARM ( $p=0.027)$. The percentage of correct chest recoil was the highest for LifeLine ARM - 100\% (95-100), 80\% (60-90) in CPRMeter group, and the lowest for manual CC - 29\% (26-48).

Conclusions: According to the results of this simulation trial, automated chest compression devices (ACCD) should be used for chest compression of patients with suspected/confirmed COVID-19. In the absence of ACCD, it seems reasonable to change the cardiopulmonary resuscitation algorithm (in the context of patients with suspected/confirmed COVID-19) by reducing the duration of the cardiopulmonary resuscitation cycle from the current 2-min to 1-min cycles due to a statistically significant reduction in the quality of chest compressions among rescuers wearing PPE AGP. (Cardiol J 2020; 27, 5: 497-506)
\end{abstract}

Key words: chest compression, cardiopulmonary resuscitation, quality, COVID-19, SARS-CoV-2, medical simulation

Address for correspondence: Lukasz Szarpak, Assoc. Prof. PhD, Lazarski University, ul. Świeradowska 43, 02-662 Warszawa, Poland, tel: +48 500186225, e-mail: lukasz.szarpak@gmail.com 


\section{Introduction}

The current coronavirus severe acute respiratory syndrome coronavirus 2 (SARS-CoV-2) pandemic which causes the disease as defined by the World Health Organization (WHO): COVID-19 represents a challenge for medical personnel, specifically including those who are particularly exposed to this type of patient [1]. Since the appearance of the first cases in China in December 2019, the virus has spread around the world. As of 1 May 2020, the number of confirmed infections worldwide has reached $3,260,373$, including 233,996 deaths from the virus. The virus is transmitted from human to human by droplets [2, 3]. Therefore, medical personnel for patients with suspected/confirmed COVID-19 should use full personal protective equipment (PPE) for aerosol generating procedures (AGP) to reduce the risk of infection [4-6]. Yang et al. [7] indicated that in COVID-19-infected patients, comorbidities and the diagnosed underlying diseases include: hypertension, respiratory system and cardiovascular diseases may be a risk factors for severe compared with a non-severe course of the disease. Considering the above, as well as a mortality rate of nearly $5.4 \%$, medical personnel may have to undertake resuscitation procedures on such a person.

Resuscitation guidelines are published by, among others, the European Resuscitation Council (ERC) $[8,9]$ or the American Heart Association (AHA) [10, 11]. On 24 April 2020 ERC published guidelines for conduct in COVID-19, which indicates the need to use personal protective equipment during resuscitation [12], but reference was not made to the impact of PPE AGE on the quality of resuscitation and thus the possibility of changing the resuscitation algorithm. However, as studies indicate, PPE may hinder medical procedures [13-15]. Chest compression systems including automatic chest compression devices (ACCD) or cardiopulmonary resuscitation (CPR) feedback devices which may be helpful in this regard. In the case of ACCD, CPR guidelines do not recommend their routine use. Resistance from the main medical community are based on the belief that ACCD causes more chest damage than manual chest compression (CC). Studies by Koster et al. [16] LUCAS suggest that a chest compression device does not cause significantly more serious or life-threatening visceral damage than manual CC.

The aim of the study was to evaluate various methods of chest compressions in a patient with suspected/confirmed SARS-CoV-2 infection conducted by medical students wearing full PPE for AGP. The hypothesis herein, is that the chest compression with LifeLine ARM was superior to CPRMeter as well as manual chest compression.

\section{Methods}

\section{Study design}

A multicenter, randomized, singe-blinded, crossover simulation study was conducted to evaluate chest compression quality of patients with suspected/confirmed COVID-19 by medical students wearing PPE for AGP. Study protocol was approved by Institutional Review Board of Polish Society of Disaster Medicine (Approval no. 09.01.2020.IRB). The study was conducted in medical simulation centers at Lazarski University (Warsaw, Poland) and Poznan University of Medical Science (Poznan, Poland) in February 2020.

\section{Participants}

The sample size was based on expected differences in time to intubation and calculated with $\mathrm{G} \times$ Power 3.1 using the two-tailed t-test (Cohen's $\mathrm{d}=0.8$, alpha error $=0.05$, power $=0.95$ ). It was determined that a minimum of 32 participants were required for a pair-wise comparison of the samples. 35 medical students were recruited who had successfully completed an advanced cardiovascular life support (ACLS) course. Written voluntary informed consent was obtained from each participant prior to the study.

\section{Equipment and materials}

Two devices were used in the present study:

- CPRMeter feedback device (Laerdal, Stavanger, Norway), which is an accelerometer device. Placed in the middle of the chest and pressed by a rescuer, it shows the correctness of the rate of chest compressions, the depth of compressions as well as chest recoil [17, 18];

- LifeLine ARM automatic chest compression device (Defibtech, LLC, Guilford, CT, USA), which allows for automatic chest compression in two modes: 30:2 and in an asynchronous mode [19].

The reference method was manual chest compression.

To simulate a patient with suspected/confirmed COVID-19 requiring CPR, Resusci Anne Advanced SkillTrainer manikin (Laerdal, Stavanger, Norway) was used, which was placed on the floor in a brightly lit room. 


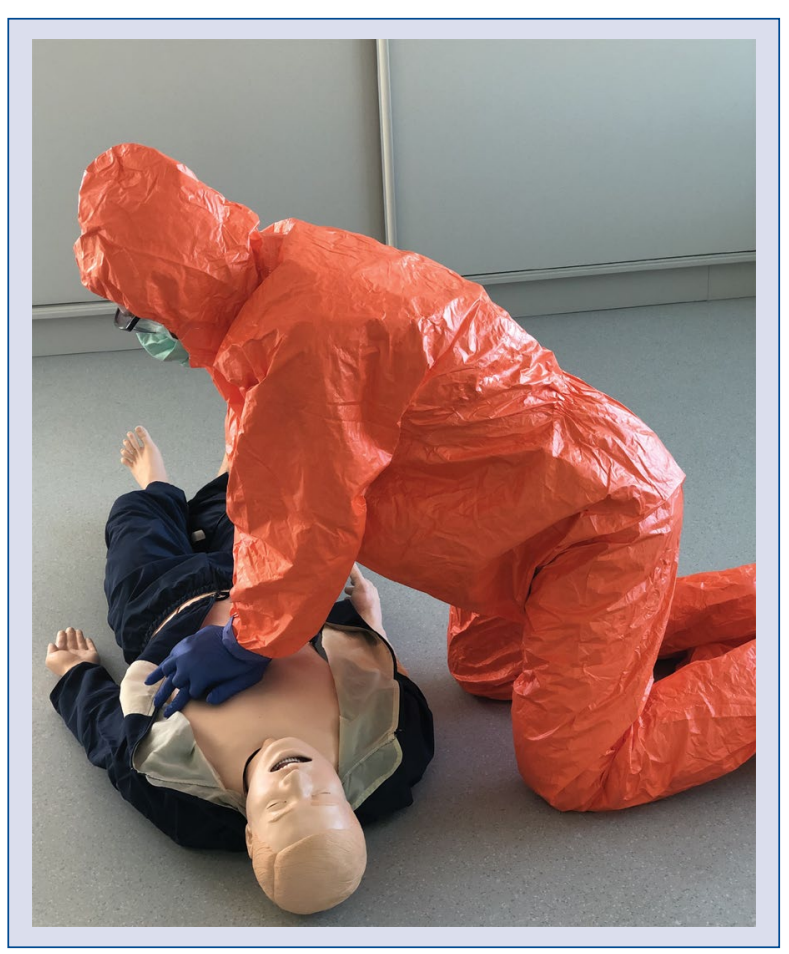

Figure 1. Rescuer with personal protective equipment for aerosol generating procedures.

The participants were dressed in a ProChem I F suit providing protection against organic and inorganic chemicals in high concentrations and against particles less than $1 \mu \mathrm{m}$ in diameter. This suit also protects against biological hazards and toxic agents and is often used during the current COVID-19 pandemic. To simulate real actions against a SARS-CoV-2 patient, the participants additionally wore a protective mask with FFP2 filter, protective goggles and a visor as well as double nitrile gloves (Fig. 1).

\section{Interventions}

All participants completed a brief questionnaire consisting of demographic information (age, sex). Before starting this trial, instructors gave medical students lectures for $30 \mathrm{~min}$ about the risks associated with SARS-CoV-2 coronavirus and how to perform CPR using the methods to be tested. The participants, wearing PPE AGP, had to conduct a 2-min cycle of continuous chest compressions in adults. In order to achieve the desired effect and focus only on parameters related to chest compressions, the scenario where the patient was intubated was foreseen, which made it possible to conduct continuous chest compressions. Chest compressions were performed using three methods:
(A) Manual CC, (B) compression using the CPRMeter feedback device, (C) compression using the LifeLine ARM system.

Both the sequence of participants and chest compression methods were random. The ResearchRandomizer program was used for this purpose. Participants were divided into three groups. The first group started compressions using the manual method, the second using CPRMeter and the third using LifeLine ARM. After a 2-min CC cycle, the participants had a 2 -h break and then performed chest compressions using another method. A detailed randomization procedure is shown in Figure 2.

\section{Measurements}

All parameters were recorded using SkillReporter software (Laerdal, Stavanger, Norway) attached to the simulator. Additionally, in order to analyze the parameters at intervals of $20 \mathrm{~s}$, the parameters were recorded in real time using GoPro HERO 5 Black camera (GoPro, Inc., CA, USA). The parameters such as: depth of CCs, rate of CCs and degree of chest recoil were analyzed. The parameters as indicated by the ERC and AHA guidelines were employed, according to the depth of CCs of an adult should be in the range of 50-60 mm, a compression rate should be from 100 to 120 compressions per minute (CPM), was used as reference values $[8,10]$.

Following the completion of this scenario, the participants were asked to grade each chest compression method based on the fatigue according to visual-analogue scale (VAS) $(1=$ no fatigued, $100=$ extremely fatigued) in the relevant scenario, but they discouraged from an overall ranking of the devices.

\section{Statistical analysis}

The data were compiled using a standard spreadsheet application (Excel, Microsoft, Redmond, WA, USA) and were analyzed using the Statistica version 13.3EN (Tibco Inc, Tulusa, OK, USA). Data were blinded from the team interpreting the results. All participant and chest compression parameter data were summarized descriptively. Categorical data are presented as raw numbers and as frequencies, and continuous and ordinal data are presented as the median and interquartile range (IQR). The Friedman test was used for intra-group analysis, and for a pairwise comparison, the Wilcoxon signed-rank test was used. In all analyses, a significance level $\mathrm{p}<0.05$ was used. 


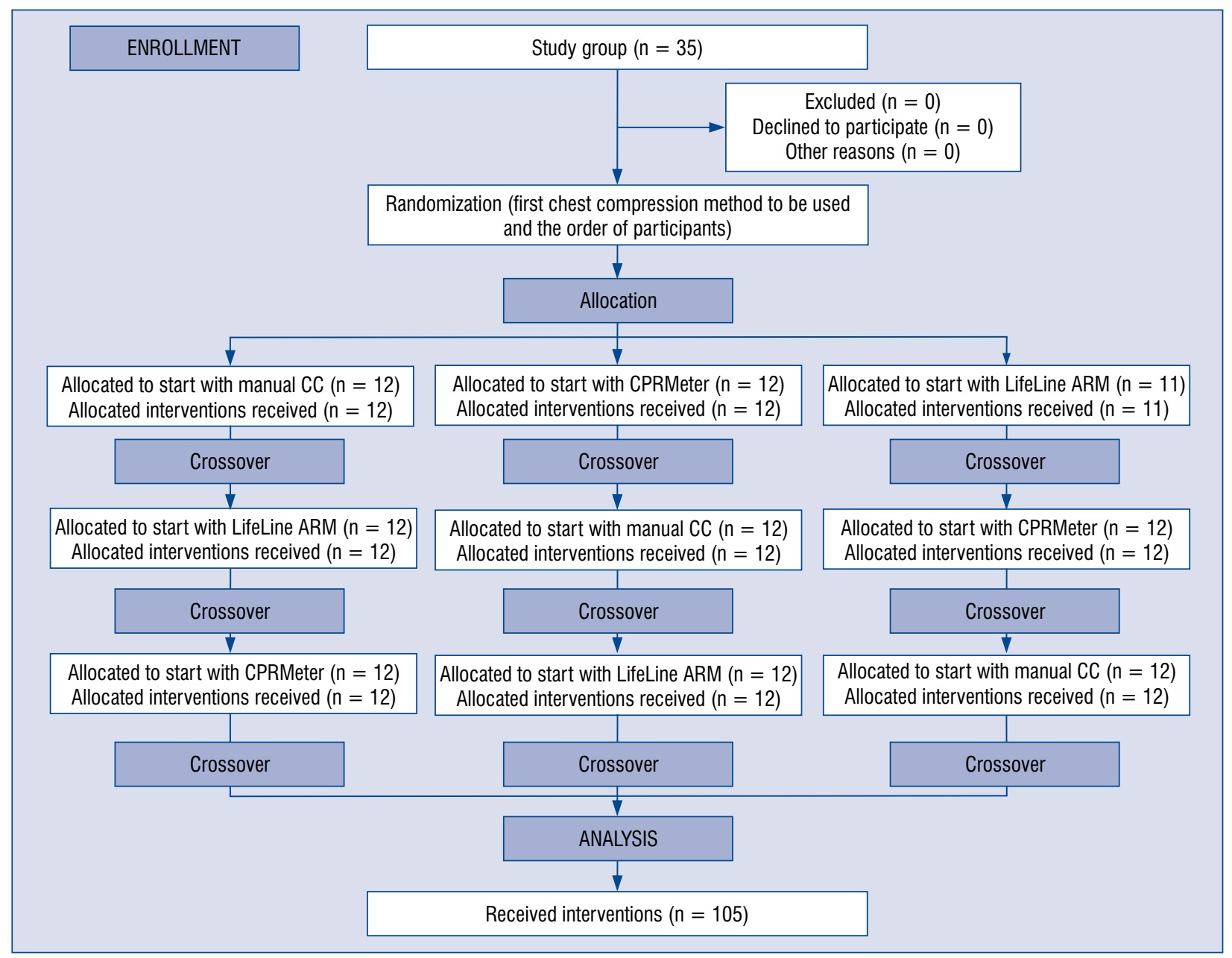

Figure 2. Randomization flow chart; CC - chest compression.

Table 1. Comparison of chest compression (CC) quality parameters.

\begin{tabular}{lcccc}
\hline Parameter & \multicolumn{3}{c}{ Chest compression technique } & P \\
\cline { 2 - 4 } & Manual CC & CPRMeter & LifeLine ARM & \\
\hline Chest compression depth & $40(38-45)$ & $45(40-50)$ & $51(50-52)$ & $\mathbf{0 . 0 0 2}$ \\
Chest compression rate & $109(102-131)$ & $107(105-127)$ & $102(101-102)$ & $\mathbf{0 . 0 2 7}$ \\
Correct chest recoil & $29(26-48)$ & $80(60-90)$ & $100(95-100)$ & $<\mathbf{0 . 0 0 1}$ \\
\hline
\end{tabular}

\section{Results}

Thirty-five medical students after an ACLS course were enrolled. There were no exclusions in the present study.

\section{Chest compression parameters}

Data on the quality of 2 -min CCs are presented in Table 1. Analysis of the quality of 2-min CCs showed statistically significant differences in the depth of CCs performed manually, using CPRMeter and LifeLine ARM (40 mm [38-45] vs. $45 \mathrm{~mm}$ [40-50] vs. $51 \mathrm{~mm}$ (50-52), respectively; $\mathrm{p}=$ $=0.002)$. Statistically significant differences in chest compression depth between manual chest compressions and CPRMeter $(\mathrm{p}=0.031)$ and LifeLine ARM $(\mathrm{p}<0.001)$ were shown. The difference was also observed between CPRMeter and LifeLine ARM ( $p=0.002$; Suppl. Table 1).

Compression rates for manual CC was 109 (IQR 102-131) CPM, 107 (IQR 105-127) CPM for CPRMeter feedback device, and 102 (IQR 101-102) 


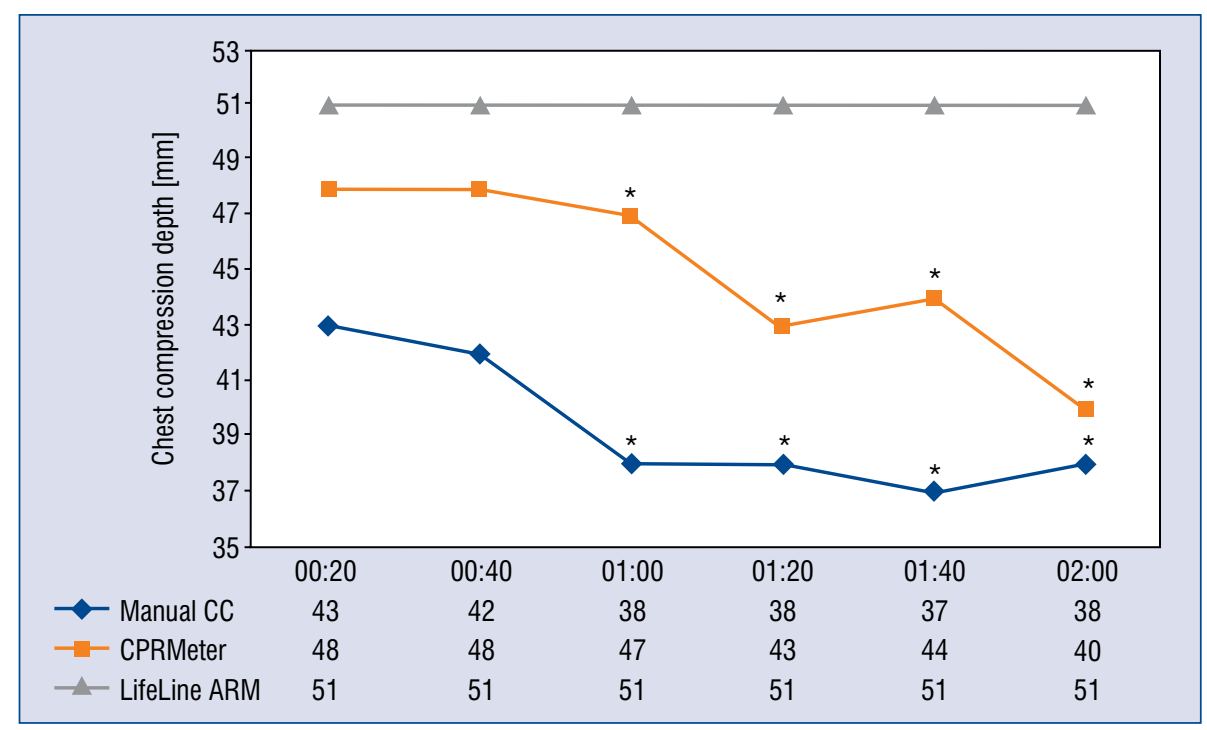

Figure 3. Chest compression (CC) depth parameters relative to time intervals; *Significant difference $(p<0.05)$ compared to the time of $20 \mathrm{~s}$ of an appropriate $\mathrm{CC}$ technique.

CPM for LifeLine ARM ( $p=0.027)$. As in the previous parameter, statistically significant differences were observed between manual compression and CPRMeter ( $\mathrm{p}=0.047$ ), manual compression and LifeLine ARM ( $p=0.001)$, and between CPRMeter and LifeLine ARM ( $\mathrm{p}=0.006)$.

The best chest recoil was observed with LifeLine ARM systems - 100\% (IQR 95-100), followed by CPRMeter - 80\% (IQR 60-90), and the lowest for manual CC $-29 \%$ (IQR 26-48). These differences were statistically significant $(\mathrm{p}<0.001)$. Two-sided analysis showed statistically significant differences in the percentage of correct chest recoils between manual $\mathrm{CC}$ and CPRMeter $(\mathrm{p}<0.001)$, manual CC vs. LifeLine ARM $(\mathrm{p}<0.001)$ as well as between CPRMeter and LifeLine ARM $(\mathrm{p}<0.001)$.

\section{Chest compression quality in 20 -s periods}

An analysis of the depth of chest compressions carried out in 20-s intervals is shown in Figure 3. Statistical analysis showed a significant reduction in the depth of CCs above $60 \mathrm{~s}$ for both manual CC and CPRMeter.

The chest compression rate showed statistically significant differences for manual $\mathrm{CC}$ and CPRMeter groups (Fig. 4).

The percentage of correct chest recoils for manual CC was significantly reduced after only $60 \mathrm{~s}$ of CPRMeter (Fig. 5). Percentage of correct chest recoils in LifeLine ARM remained the same throughout the entire chest compression period.

\section{Fatigue VAS score}

The degree of fatigue of study participants performing $\mathrm{CCs}$ based on VAS score when using manual CC, CPRMeter and LifeLine ARM groups was varied and were observed accordingly 75 (IQR 45-90) vs. 80 (IQR 50-90) vs. 20 (IQR $20-30)$ points $(\mathrm{p}=0.002)$. There was statistically significant differences in degree of fatigue between manual chest compression and LifeLine ARM ( $\mathrm{p}<0.001$ ), and between CPRMeter and LifeLine ARM $(\mathrm{p}<0.001)$.

\section{Discussion}

Recent guidelines of the ERC as well as the AHA indicate a direct impact of high-quality $\mathrm{CC}$ on the effectiveness of resuscitation $[8,10]$ and thus, the return of spontaneous circulation and reduction of neurological deficits caused by hypoxemia.

During CPR, the need to interrupt CCs to provide synchronous ventilation prevents blood flow continuity, reducing the possibility to ensure high-quality CPR and have a negative impact on perfusion and patient outcome [20,21]. In this study, continuous CCs were performed because, as indicated by ERC and AHA guidelines, the key role during CPR is to minimize pauses in CCs $[8,10]$. In the case of patient intubation, continuous (asynchronous to emergency ventilation) $\mathrm{CCs}$ are possible [22]. As numerous studies indicate, it is the most effective method, because by eliminating long pauses accompanying rescue breathing improves 


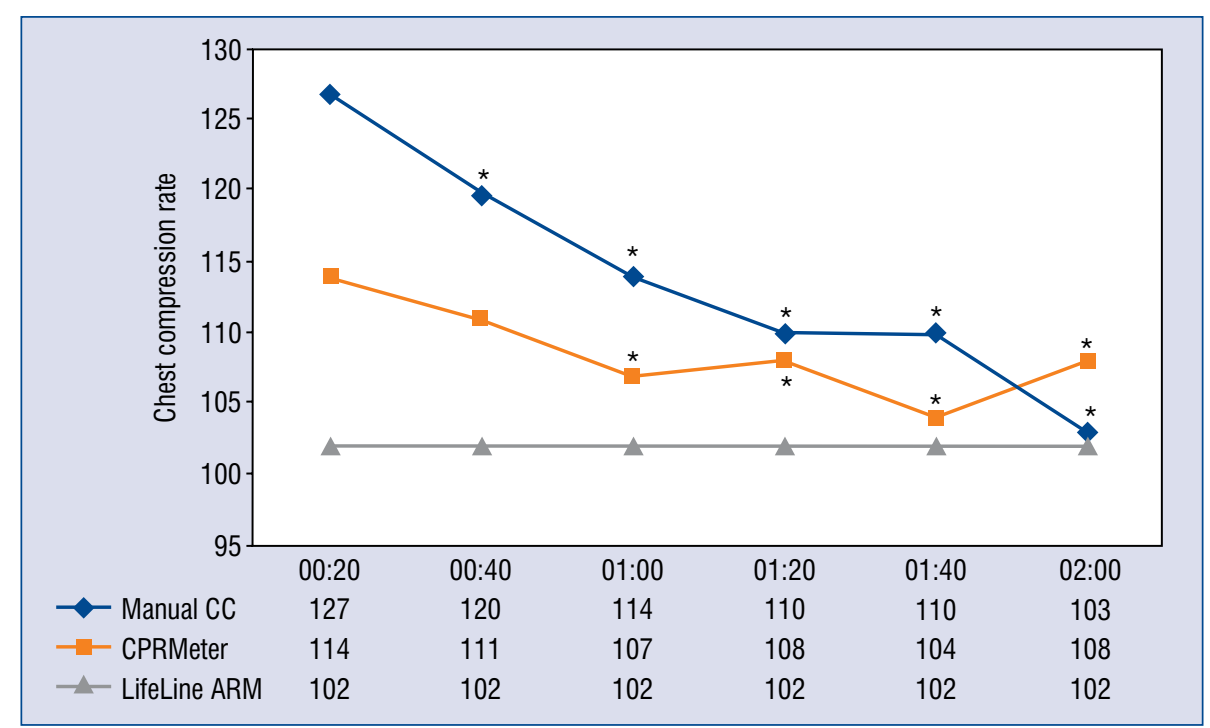

Figure 4. Chest compression (CC) rate parameters relative to time intervals; *Significant difference $(p<0.05)$ compared to the time of $20 \mathrm{~s}$ of an appropriate CC technique.

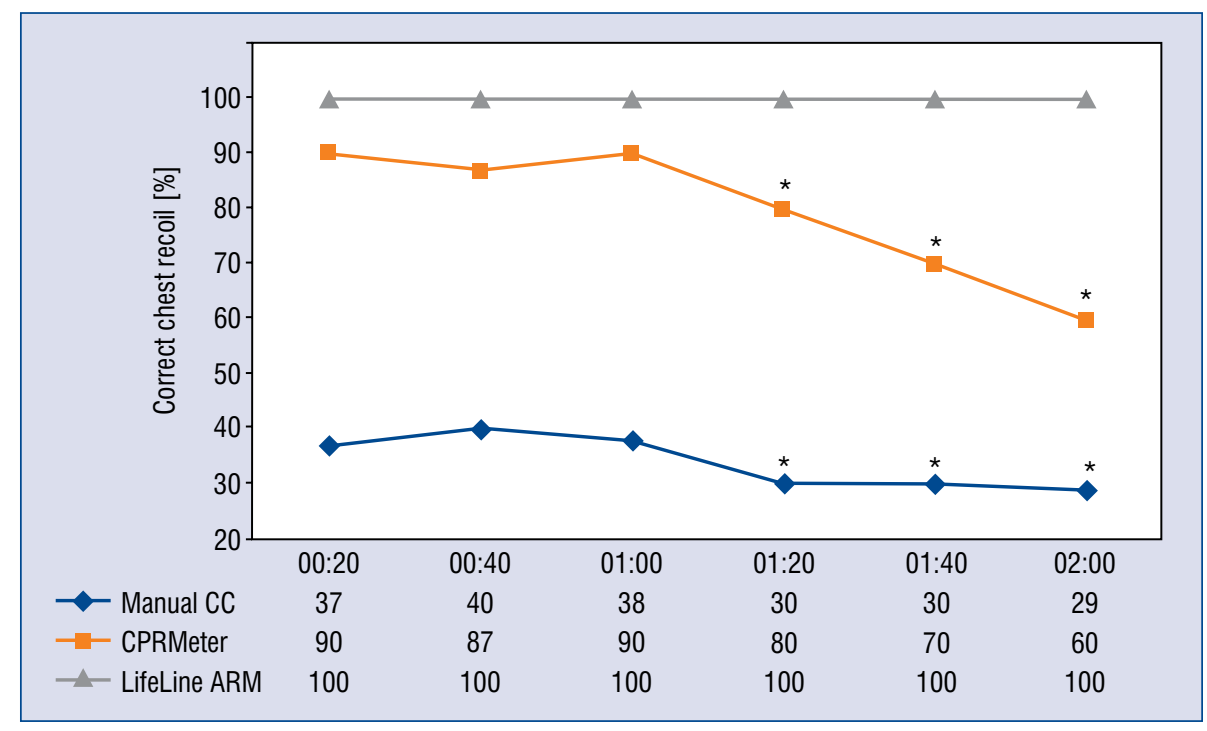

Figure 5. Percentage of correct chest recoil parameters relative to time intervals; ${ }^{*}$ Significant difference $(p<0.05)$ compared to the time of $20 \mathrm{~s}$ of an appropriate chest compression (CC) technique.

perfusion pressure [22-24]. Continuous chest compression, as indicated by Heidenreich et al. [23] resulted in more adequate compressions per minute than standard CPR for the first 2 min of CPR. However, as the duration of the resuscitation increases, continuous chest compression technique leads to more fatigue for the rescuer. The reduction of fatigue may be influenced by the physical condition of the rescuer [25]. However, the application of PPE, as shown by numerous studies, may reduce the efficiency of medical procedures [26], starting with CCs [27], by obtaining vascular access [28, $29]$, ending with airway management [30, 31].

A factor influencing the quality of CPR is the depth of CCs [32]. For CPR without PPE AGP, the depth of CCs decreases after about 2 min of compressions [33].

In the current study there were statistically significant differences in the depth of CCs between the different methods of CCs. In the case of manual 
CC and CPRMeter groups, a statistically significant decrease in the depth of CCs was observed after 1 min of resuscitation, which may have been caused by excessive fatigue of participants performing CCs as a result of using PPE AGP [13]. Other authors also point to the problem of reduced quality of $\mathrm{CCs}$ when using PPE [13, 27, 34]. In the present study, the CC depth during the use of ACCD was equal throughout the whole resuscitation process and was consistent with current CPR guidelines, due to the fact that the chest $\mathrm{CC}$ depth was performed automatically. This method of compression also allows CPR to be performed during patient transport to the hospital as well as during prolonged resuscitation [35, 36].

During CPR full chest recoil after each compression is independently associated with improved survival and is independently associated with improved survival and favorable neurologic outcome at hospital discharge after adult out-of-hospital cardiac arrest [37, 38]. Analysis of the obtained results showed that medical students dressed in PPE AGP perform manual CCs in an insufficient manner. The problem of incomplete chest relaxation is reduced when using CPRMeter. The results obtained are confirmed by other studies $[39,40]$. Similar to the depth of compressions, chest recoil is significantly reduced after $1 \mathrm{~min}$ of continuous CC (in manual CC and CPRMeter groups). This may be due to fatigue of the rescuer and subsequent $\mathrm{CC}$ after each compression. $\mathrm{CCs}$ to the appropriate depth and then performing full chest recoil is a prerequisite for optimal perfusion pressure [24, 41].

The rate of $\mathrm{CC}$ is also an important element of high-quality CC. CPR guidelines recommend that CC should be performed at a rate of 100-120 CPM. Idris et al. [42] confirms that compression rates between 100 and 120 per minute were associated with the greatest survival to hospital discharge. A higher compression rate than $120 \mathrm{CPM}$ may improve organ perfusion but does not increase survival. However, it may lead to faster fatigue of the rescuer, which consequently results in lower quality of CCs [43, 44]. Chen at al. [34] suggested that the use of PPE may reduce the rate of chest compression.

Cardiopulmonary resuscitation feedback devices facilitate $\mathrm{CC}$ s by showing real-time compression parameters [45, 46]. Iskrzycki et al. [47] in his study showed that visual real-time feedback device significantly improved quality of CPR performer by lifeguards. In contrast Wattenbarger et al. [48] stated that a targeted training intervention combined with real-time CPR feedback improved CC performance among health care providers. However, the use of such a device still requires force from the rescuer and also leads to fatigue. In the study, after 1 min of continuous CCs, rescuers dressed in PPE AGP were both statistically significant in reducing the depth of CCs and in reducing chest recoil. This may result in reduced effectiveness of the whole resuscitation process. Another solution aimed at improving the quality of $\mathrm{CC}$ is the automatic $\mathrm{CC}$ system. Taking into account the fact that the quality of CCs performed by medical personnel is in many cases insufficient [49], there can be a remedy for this problem. Analysis of the data obtained in this study showed that LifeLine ARM, an example of ACCD, performed CCs at the appropriate depth and at the programmed compression rate. As indicated by the studies Szarpak et al. [50], and Truszewski et al. [51] LifeLine ARM resuscitation using LifeLine ARM had significantly better quality compared to manual chest compressions.

The use of such systems is particularly important when paramedics are unable to perform high quality CPR - and this is the case for patients with suspected/confirmed COVID-19 when, due to the coronavirus, personnel must be equipped with PPE AGP.

\section{Limitations of the study}

There were several limitations in the present study. First, an adult manikin was used to simulate patients requiring CPR. Therefore, the quality of chest compressions may differ from that of CPR under real CPR. However, the choice of medical simulation as a research method was deliberate and was dictated by the fact that it is medical simulation that allows for full standardization of performed procedures without the risk of complications for a potential patient $[24,52,53]$, moreover, in the current pandemic, conducting research - in particular randomized cross-over study under emergency conditions could endanger both the patient and the rescuer. The second limitation was to include only medical students in the study, however, this group may be involved in providing medical assistance in a disaster or emergency situation, hence an assessment of the possibility of CPR in PPE AGP is one of the key actions to determine an optimal method of CPR.

The study also has its strengths. Among them, was the randomized cross-over study design, as well as the fact that it was a multi-center study. Additionally, a single-blinded study was utilized, increasing its value. Another aspect supporting 
this study is the fact that, according to available research, this is the first study comparing different methods of $\mathrm{CC}$ of patients with suspected/ /confirmed COVID-19 by rescuers wearing personal protective equipment for aerosol generating procedures.

\section{Conclusions}

In conclusion, according to the results of this simulation trial, ACCD should be used for CC of patients with suspected/confirmed COVID-19. In the absence of ACCD, it seems reasonable to change the CPR algorithm (in the context of patients with suspected/confirmed COVID-19) by reducing the duration of the CPR cycle for one rescuer from the current 2-min to 1-min cycles due to a statistically significant reduction in the quality of CCs among rescuers wearing PPE AGP. More studies on chest compression quality with PPE AGP should be conducted to confirm those data.

\section{Acknowledgements}

We would thank to all the medical students who participated in this trial. The present study was supported by the ERC Research NET and Polish Society of Disaster Medicine.

Conflict of interest: Bernd W. Böttiger is European Resuscitation Council (ERC) Board Director Science and Research; Chairman of the German Resuscitation Council (GRC); Member of the, Advanced Life Support (ALS) Task Force of the International Liaison Committee on Resuscitation, Member of the executive committee of the German Interdisciplinary Association for Intensive and Emergency Medicine (DIVI); Associated Editor of the European Journal of Anaesthesiology (EJA), Co-Editor of "Resuscitation"; Editor of the Journal "Notfall + Rettungsmedizin". He received professional fees for lectures from the following companies: Medupdate $\mathrm{GmbH}$, "Forum für medizinische Fortbildung (FomF)", Baxalta Deutschland GmbH, Bayer Vital GmbH, ZOLL Medical Deutschland GmbH, C.R. Bard GmbH, GS Elektromedizinische Geräte G. Stemple GmbH. Others authors have no potential conflict of interest relevant to this article.

\section{References}

1. Rothan HA, Byrareddy SN. The epidemiology and pathogenesis of coronavirus disease (COVID-19) outbreak. J Autoimmun. 2020; 109: 102433, doi: 10.1016/j.jaut.2020.102433, indexed in Pubmed: 32113704.
2. van Doremalen N, Bushmaker T, Morris DH, et al. Aerosol and surface stability of SARS-CoV-2 as compared with SARS-CoV-1. N Engl J Med. 2020; 382(16): 1564-1567, doi: 10.1056/ NEJMc2004973, indexed in Pubmed: 32182409.

3. Dzieciatkowski T, Szarpak L, Filipiak KJ, et al. COVID-19 challenge for modern medicine. Cardiol J. 2020; 27(2): 175-183, doi: 10.5603/CJ.a2020.0055, indexed in Pubmed: 32286679.

4. Verbeek JH, Rajamaki B, Ijaz S, et al. Personal protective equipment for preventing highly infectious diseases due to exposure to contaminated body fluids in healthcare staff. Cochrane Database Syst Rev. 2016; 4: CD011621, doi: 10.1002/14651858. CD011621.pub2, indexed in Pubmed: 27093058.

5. Smereka J, Szarpak L. COVID 19 a challenge for emergency medicine and every health care professional. Am J Emerg Med. 2020 [Epub ahead of print], doi: 10.1016/j.ajem.2020.03.038, indexed in Pubmed: 32241630.

6. Smereka J, Szarpak L, Filipiak K. Modern medicine in COVID-19 era. Disaster Emerg Med J. 2020, doi: 10.5603/demj.a2020.0012.

7. Yang J, Zheng Ya, Gou Xi, et al. Prevalence of comorbidities and its effects in coronavirus disease 2019 patients: A systematic review and meta-analysis. Int J Infect Dis. 2020 [Epub ahead of print]; 94: 91-95, doi: 10.1016/j.ijid.2020.03.017, indexed in Pubmed: 32173574.

8. Soar J, Nolan JP, Böttiger BW, et al. Adult advanced life support section Collaborators. European Resuscitation Council Guidelines for Resuscitation 2015: Section 3. Adult advanced life support. Resuscitation. 2015; 95: 100-147, doi: 10.1016/j.resuscitation.2015.07.016, indexed in Pubmed: 26477701.

9. Truhlár A, Deakin CD, Soar J, et al. Cardiac arrest in special circumstances section Collaborators. European Resuscitation Council Guidelines for Resuscitation 2015: Section 4. Cardiac arrest in special circumstances. Resuscitation. 2015; 95: 148-201, doi: 10.1016/j. resuscitation.2015.07.017, indexed in Pubmed: 26477412.

10. Link MS, Berkow LC, Kudenchuk PJ, et al. Part 7: Adult Advanced Cardiovascular Life Support: 2015 American Heart Association Guidelines Update for Cardiopulmonary Resuscitation and Emergency Cardiovascular Care. Circulation. 2015; 132(18 Suppl 2): S444-S464, doi: 10.1161/CIR.0000000000000261, indexed in Pubmed: 26472995.

11. Lavonas EJ, Drennan IR, Gabrielli A, et al. Part 10: Special Circumstances of Resuscitation: 2015 American Heart Association Guidelines Update for Cardiopulmonary Resuscitation and Emergency Cardiovascular Care. Circulation. 2015; 132(18 Suppl 2): S501-S518, doi: 10.1161/CIR.0000000000000264, indexed in Pubmed: 26472998.

12. European Resuscitation Council. European Resuscitation Council COVID-19 Guidelines. www.erc.edu (Access: 24 April, 2020).

13. Martín Rodríguez F, Fernández Pérez C, Castro Villamor M, et al. Does level D personal protective equipment guard against hazardous biologic agents during cardiopulmonary resuscitation? Emergencias. 2018; 30(2): 119-122, indexed in Pubmed: 29547235.

14. Wiechmann W, Toohey S, Majestic C, et al. Intubating ebola patients: technical limitations of extensive personal protective equipment. West J Emerg Med. 2015; 16(7): 965, doi: 10.5811/ westjem.2015.10.28779, indexed in Pubmed: 26759639.

15. Smereka J, Szarpak L, Filipiak KJ, et al. Which intravascular access should we use in patients with suspected/confirmed COVID-19? Resuscitation. 2020 [Epub ahead of print]; 151: 8-9, doi: 10.1016/j.resuscitation.2020.04.014, indexed in Pubmed: 32304800 . 
16. Koster RW, Beenen LF, van der Boom EB, et al. Safety of mechanical chest compression devices AutoPulse and LUCAS in cardiac arrest: a randomized clinical trial for non-inferiority. Eur Heart J. 2017; 38(40): 3006-3013, doi: 10.1093/eurheartj/ehx318, indexed in Pubmed: 29088439.

17. Iskrzycki L, Smereka J, Rodriguez-Nunez A, et al. The impact of the use of a CPRMeter monitor on quality of chest compressions: a prospective randomised trial, cross-simulation. Kardiol Pol. 2018; 76(3): 574-579, doi: 10.5603/KP.a2017.0255, indexed in Pubmed: 29297195.

18. Majer J, Kaminska H, Wieczorek W, et al. Impact of a cprmeter feedback device on chest compression quality performed by nurses - a randomized crossover study. Disaster Emerg Med J. 2018; 3(1): 36-37, doi: 10.5603/demj.2018.0008.

19. Szarpak L, Truszewski Z, Czyzewski L, et al. CPR using the lifeline ARM mechanical chest compression device: a randomized, crossover, manikin trial. Am J Emerg Med. 2017; 35(1): 96-100, doi: 10.1016/j.ajem.2016.10.012, indexed in Pubmed: 27756513.

20. Ewy GA, Zuercher M, Hilwig RW, et al. Improved neurological outcome with continuous chest compressions compared with 30:2 compressions-to-ventilations cardiopulmonary resuscitation in a realistic swine model of out-of-hospital cardiac arrest. Circulation. 2007; 116(22): 2525-2530, doi: 10.1161/CIRCULATIONAHA.107.711820, indexed in Pubmed: 17998457.

21. Smereka J, Bielski K, Ladny JR, et al. Evaluation of a newly developed infant chest compression technique: A randomized crossover manikin trial. Medicine (Baltimore). 2017; 96(14): e5915, doi: 10.1097/MD.0000000000005915, indexed in Pubmed: 28383397.

22. Heidenreich JW, Berg RA, Higdon TA, et al. Rescuer fatigue: standard versus continuous chest-compression cardiopulmonary resuscitation. Acad Emerg Med. 2006; 13(10): 1020-1026, doi: 10.1197/j.aem.2006.06.049, indexed in Pubmed: 17015418.

23. Heidenreich JW, Sanders AB, Higdon TA, et al. Uninterrupted chest compression CPR is easier to perform and remember than standard CPR. Resuscitation. 2004; 63(2): 123-130, doi: 10.1016/j.resuscitation.2004.04.011, indexed in Pubmed: 15531062

24. Smereka J, Szarpak L, Rodríguez-Núñez A, et al. A randomized comparison of three chest compression techniques and associated hemodynamic effect during infant CPR: A randomized manikin study. Am J Emerg Med. 2017; 35(10): 1420-1425, doi: 10.1016/j.ajem.2017.04.024, indexed in Pubmed: 28433454.

25. Abelairas-Gómez C, Barcala-Furelos R, Szarpak $€$, et al. The effect of strength training on quality of prolonged basic cardiopulmonary resuscitation. Kardiol Pol. 2017; 75(1): 21-27, doi: 10.5603/KP.a2016.0165, indexed in Pubmed: 27878801.

26. Adler MD, Krug S, Eiger C, et al. Impact of personal protective equipment on the performance of emergency pediatric tasks. Pediatr Emerg Care. 2020 [Epub ahead of print], doi: 10.1097/ PEC.0000000000002028, indexed in Pubmed: 32097378.

27. Szarpak L, Truszewski Z, Gałązkowski R, et al. Comparison of two chest compression techniques when using CBRN-PPE: a randomized crossover manikin trial. Am J Emerg Med. 2016; 34(5): 913-915, doi: 10.1016/j.ajem.2016.02.029, indexed in Pubmed: 26947371.

28. Szarpak L, Truszewski Z, Smereka J, et al. Comparison of two intravascular access techniques when using CBRN-PPE: A randomized crossover manikin trial. Am J Emerg Med. 2016; 34(6): 1170-1172, doi: 10.1016/j.ajem.2016.03.046, indexed in Pubmed: 27066934 .
29. Lamhaut L, Dagron C, Apriotesei R, et al. Comparison of intravenous and intraosseous access by pre-hospital medical emergency personnel with and without $\mathrm{CBRN}$ protective equipment. Resuscitation. 2010; 81(1): 65-68, doi: 10.1016/j.resuscitation.2009.09.011, indexed in Pubmed: 19854554.

30. Schumacher J, Arlidge J, Garnham F, et al. A randomised crossover simulation study comparing the impact of chemical, biologi$\mathrm{cal}$, radiological or nuclear substance personal protection equipment on the performance of advanced life support interventions. Anaesthesia. 2017; 72(5): 592-597, doi: 10.1111/anae.13842, indexed in Pubmed: 28251618.

31. Castle N, Pillay Y, Spencer N. Comparison of six different intubation aids for use while wearing CBRN-PPE: a manikin study. Resuscitation. 2011; 82(12): 1548-1552, doi: 10.1016/j.resuscitation.2011.06.020, indexed in Pubmed: 21763057.

32. Tranberg T, Lassen JF, Kaltoft AK, et al. Quality of cardiopulmonary resuscitation in out-of-hospital cardiac arrest before and after introduction of a mechanical chest compression device, LUCAS-2; a prospective, observational study. Scand J Trauma Resusc Emerg Med. 2015; 23: 37, doi: 10.1186/s13049-015-0114-2, indexed in Pubmed: 25898992.

33. Enriquez D, Firenze L, Fernández Díaz J, et al. Changes in the depth of chest compressions during cardiopulmonary resuscitation in a pediatric simulator. Arch Argent Pediatr. 2018; 116(6): e730-e735, doi: 10.5546/aap.2018.eng.e730, indexed in Pubmed: 30457721.

34. Chen J, Lu KZ, Yi B, et al. Chest compression with personal protective equipment during cardiopulmonary resuscitation: a randomized crossover simulation study. Medicine (Baltimore). 2016; 95(14): e3262, doi: 10.1097/MD.0000000000003262, indexed in Pubmed: 27057878.

35. Gates S, Quinn T, Deakin CD, et al. Mechanical chest compression for out of hospital cardiac arrest: Systematic review and meta-analysis. Resuscitation. 2015; 94: 91-97, doi: 10.1016/j. resuscitation.2015.07.002, indexed in Pubmed: 26190673.

36. Eichhorn S, Mendoza A, Prinzing A, et al. Corpuls CPR generates higher mean arterial pressure than LUCAS II in a pig model of cardiac arrest. Biomed Res Int. 2017; 2017: 5470406, doi: 10.1155/2017/5470406, indexed in Pubmed: 29392137.

37. Kovacs A, Vadeboncoeur TF, Stolz U, et al. Chest compression release velocity: Association with survival and favorable neurologic outcome after out-of-hospital cardiac arrest. Resuscitation. 2015; 92: 107-114, doi: 10.1016/j.resuscitation.2015.04.026, indexed in Pubmed: 25936931.

38. Smereka J, Madziala M, Szarpak L. Comparison of two infant chest compression techniques during simulated newborn cardiopulmonary resuscitation performed by a single rescuer: A randomized, crossover multicenter trial. Cardiol J. 2019; 26(6): 761-768, doi: 10.5603/CJ.a2018.0090, indexed in Pubmed: 30155866.

39. Kurowski A, Szarpak $€$, Bogdański $€$, et al. Comparison of the effectiveness of cardiopulmonary resuscitation with standard manual chest compressions and the use of TrueCPR and PocketCPR feedback devices. Kardiol Pol. 2015; 73(10): 924-930, doi: 10.5603/KP.a2015.0084, indexed in Pubmed: 25985725.

40. Majer J, Jaguszewski MJ, Frass M, et al. Does the use of cardiopulmonary resuscitation feedback devices improve the quality of chest compressions performed by doctors? A prospective, randomized, cross-over simulation study. Cardiol J. 2019; 26(5): 529535, doi: 10.5603/CJ.a2018.0091, indexed in Pubmed: 30155865.

41. Lampe JW, Tai Y, Bratinov G, et al. Developing a kinematic understanding of chest compressions: the impact of depth and 
Cardiology Journal 2020, Vol. 27, No. 5

release time on blood flow during cardiopulmonary resuscitation. Biomed Eng Online. 2015; 14: 102, doi: 10.1186/s12938-0150095-4, indexed in Pubmed: 26537881.

42. Idris AH, Guffey D, Pepe PE, et al. Chest compression rates and survival following out-of-hospital cardiac arrest. Crit Care Med. 2015; 43(4): 840-848, doi: 10.1097/CCM.0000000000000824, indexed in Pubmed: 25565457.

43. Monsieurs KG, De Regge M, Vansteelandt K, et al. Excessive chest compression rate is associated with insufficient compression depth in prehospital cardiac arrest. Resuscitation. 2012; 83(11): 1319-1323, doi: 10.1016/j.resuscitation.2012.07.015, indexed in Pubmed: 22828356.

44. Smereka J, Iskrzycki $Ł$, Makomaska-Szaroszyk E, et al. The effect of chest compression frequency on the quality of resuscitation by lifeguards. A prospective randomized crossover multicenter simulation trial. Cardiol J. 2019; 26(6): 769-776, doi: 10.5603/CJ.a2018.0121, indexed in Pubmed: 30338845.

45. Smereka J, Szarpak L, Czekajlo M, et al. The TrueCPR device in the process of teaching cardiopulmonary resuscitation: A randomized simulation trial. Medicine (Baltimore). 2019; 98(27): e15995, doi: 10.1097/MD.0000000000015995, indexed in Pubmed: 31277091.

46. Truszewski Z, Szarpak L, Kurowski A, et al. Randomized trial of the chest compressions effectiveness comparing 3 feedback CPR devices and standard basic life support by nurses. Am J Emerg Med. 2016; 34(3): 381-385, doi: 10.1016/j.ajem.2015.11.003, indexed in Pubmed: 26612703.
47. Iskrzycki L, Smereka J, Rodriguez-Nunez A, et al. The impact of the use of a CPRMeter monitor on quality of chest compressions: a prospective randomised trial, cross-simulation. Kardiol Pol. 2018; 76(3): 574-579, doi: 10.5603/KP.a2017.0255, indexed in Pubmed: 29297195.

48. Wattenbarger S, Silver A, Hoyne T, et al. Real-Time cardiopulmonary resuscitation feedback and targeted training improve chest compression performance in a cohort of international healthcare providers. J Emerg Med. 2019 [Epub ahead of print], doi: 10.1016/j.jemermed.2019.09.027, indexed in Pubmed: 31708314.

49. Wagner M, Bibl K, Hrdliczka E, et al. Effects of feedback on chest compression quality: a randomized simulation study. Pediatrics. 2019; 143(2), doi: 10.1542/peds.2018-2441, indexed in Pubmed: 30700565.

50. Szarpak L, Filipiak KJ, Ładny JR, et al. Should nurses use mechanical chest compression devices during CPR? Am J Emerg Med. 2016; 34(10): 2044-2045, doi: 10.1016/j.ajem.2016.07.057, indexed in Pubmed: 27528048.

51. Truszewski Z, Szarpak L, Kurowski A, et al. Mechanical chest compression with the LifeLine ARM device during simulated CPR. Am J Emerg Med. 2016; 34(5): 917, doi: 10.1016/j. ajem.2016.02.025, indexed in Pubmed: 26924321.

52. Abelsson A. Learning through simulation. Disaster Emerg Med J. 2017; 2(3): 125-128, doi: 10.5603/demj.2017.0027.

53. Adamczuk J, Nieckula M, Dabrowska A, et al. Recommendations for the use of simulation methods in a selected area of health sciences based on an example simulation scenario. Disaster Emerg Med J. 2019; 4(4): 173-179, doi: 10.5603/demj.a2019.0031. 